\title{
Do Underlying Measures of Inflation Outperform Headline Rates? Evidence from Australian Data*
}

\author{
Lei Lei Song \\ Melbourne Institute of Applied Economic and Social Research \\ The University of Melbourne
}

Melbourne Institute Working Paper No. 29/03

ISSN 1328-4991 (Print)

ISSN 1447-5863 (Online)

ISBN 0734031424

December 2003

*The author would like to thank the Faculty of Economics and Commerce for financial support. All errors are the responsibility of the author.

Melbourne Institute of Applied Economic and Social Research

The University of Melbourne

Victoria 3010 Australia

Telephone (03) 83445330

Fax (03) 83445630

Email melb-inst@unimelb.edu.au

WWW Address http://www.melbourneinstitute.com 


\begin{abstract}
Many central banks often focus on underlying measures of inflation when assessing inflation trends. This paper compares the accuracy of underlying measures of inflation relative to the headline rates by using Australian data. It is found that the underlying measures did have smaller errors in predicting the long-term trend in inflation than the quarterly headline rate in the sample period 1977-2001 and the inflation targeting subperiod starting from 1993, due to the large volatility of the headline rate. As compared to the year-ended headline rate, the statistical test results, however, support only the measure of market prices excluding volatile items, not the others. There is some weak evidence of the weighted median measure outperforming the headline rate in the subperiod after 1993. With respect to directional accuracy, the test statistics cannot reject the null hypothesis of an equal probability correctly predicting the moving direction of the inflation trend, though the headline rates have a higher probability.
\end{abstract}




\section{Introduction}

Accurate and reliable measures of inflation have become increasingly important as the focus of monetary policy in many countries shifts to explicit inflation targeting. Since the aggregate price index in an economy is often affected by short-run movements in some volatile components, such as fruit and fuel, it is difficult for policy makers to determine whether a change in the aggregate price index reflects movements in the fundamental determinants of inflation and also how persistent the change is likely to be. As a result, a number of central banks focus on underlying (or core) measures to help determine the outlook of inflation. The idea is that these measures may provide a better indicator of long-term trends in inflation. ${ }^{1}$

There are two types of underlying measures: exclusion (such as the CPI excluding food and energy), and statistical (such as the weighted median). In Australia, although the inflation target is expressed in terms of the consumer price index (CPI), the Reserve Bank of Australia believes that 'it can be useful when assessing inflationary trends to focus on underlying measures which abstract from short-run volatility'.2 ${ }^{2}$ These underlying measures are the 'CPI excluding volatile items', ${ }^{3}$ the 'market goods and services excluding volatile items', the '(30 per cent) trimmed mean' and the 'weighted median'.

As central banks adopts underlying measures of inflation, economists have started to evaluate these measures. Freeman (1998) tests the information contents of core inflation measures on US data by using Granger causality tests in forecasting the headline rate. While considering a wider set of underlying measures, Le Bihan and Sedillot (2000) compare the accuracy of these measures in forecasting the headline inflation rate of France. The authors of these two papers seem to give some empirical support to statistical measures.

\footnotetext{
${ }^{1}$ Wynne (1999) provides a critical review of the literature on measuring core inflation, and Cecchetti and Groshen (2000) surveys the measurement of underlying inflation.

${ }^{2}$ See Box D in Reserve Bank of Australia (2002). In RBA's reports, the long-term trend in inflation is usually called the 'underlying trend of inflation'. To avoid the confusion between underlying trends and underlying measures, this paper uses 'the long-term trend in inflation' to replace the term of 'the underlying inflation trend'.

${ }^{3}$ Volatile items are defined as fruit, vegetables and automotive fuel by the Australian Bureau of Statistics.
} 
After constructing an approximation of the long-term trend in inflation for the US, Cecchetti (1997) examines the efficiency (small sample variance) of various inflation measures by using a Monte Carlo experiment based on actual data. When comparing monthly changes of the measures, he finds that a trimmed mean measure is the most efficient estimator. With respect to deviations from the long-term trend in inflation, his results show that the headline rate averaged over 12 months does have a slightly bigger confidence interval than the 10 per cent trimmed mean, but smaller than all the other alternative measures.

By conducting a series of trials for trimmed means, Kearns (1998) finds that the 100 per cent trim (i.e., the weighted median) is the best trimmed mean measure of inflation in Australia. Dixon and Lim (2003) evaluate various underlying measures used by Australian authorities against a set of criteria about their time series relationship with the headline rate, such as cointegration and Granger causation. They conclude that the majority of the underlying measures are not satisfactory in that they are excluding useful information.

From a different perspective, this paper compares the performance of the CPI headline rates and underlying measures in Australia by assessing their relative accuracy in predicting the long-term trend in inflation. The paper is structured as follows. Section 2 provides a simple framework for analysis, while Section 3 briefly introduces the alternative indicators and presents the methods to assess their performance. Section 4 reports the relative performance of the various underlying measures with respect to the headline rates. Section 5 concludes the paper.

\section{A framework for analysis}

Between any two periods, the changes in the prices of individual goods and services have two elements, one is common to all goods and services that constitutes the long-term trend in inflation (or sometimes called core or underlying inflation in the literature), and the other is idiosyncratic for the local market. The problem in measuring the long-term 
trend is to isolate these two elements from observed price changes. Define $\dot{p}_{i, t}$ as the rate of change of an individual price $i$ at time $t, \Pi_{t}=\ln \left(P_{t} / P_{t-1}\right)$ as an aggregate inflation component and $x_{i, t}$ a relative price change element, this can be written as

$$
\dot{p}_{i, t}=\Pi_{t}+x_{i, t}
$$

$\Pi$ is the object of interest. This is the long-term trend in all prices and is therefore the target of monetary policy.

The 'headline' rate is normally constructed as a weighted average of individual price changes, $\pi_{t}=\sum_{i} w_{i, t} \dot{p}_{i, t}$, where $\sum_{i} w_{i, t}=1$. The weights are usually chosen on the basis of expenditure shares. Since $\Pi_{t}$ is common to all prices, Equation 1 implies

$$
\pi_{t}-\Pi_{t}=\sum_{i} w_{i, t} x_{i, t}
$$

The term on the right side of the equation is usually nonzero and as a result the headline rate in any period is a poor approximation to the long-term trend. Following the literature, ${ }^{4}$ this term can be decomposed into two parts, noise $\left(n_{t}\right)$ and bias $\left(b_{t}\right)$. The noise is transitory and mean zero, implying that lengthening the observation interval would eliminate this transitory noise. The bias, however, is permanent with a nonzero mean, which is normally the consequence of weighting patterns, sampling techniques, and quality adjustment employed in the calculation of the headline rate. Those biases that result from actual errors in measuring the individual prices themselves can only be dealt with in the compilation procedure, and are outside the scope of this paper.

Lengthening the observation interval over which inflation is measured, such as averaging headline rates over many months and years, will gradually reduce noise, but this will certainly decrease the usefulness to central bankers, who have to wait for noise to be eliminated over time. Various underlying measures of inflation are therefore designed to provide as much information on the long-term trend as possible from timely released price indices. These measures minimise the transitory noise by using different informa-

\footnotetext{
${ }^{4}$ See, for example, Boskin and et al. (1997) and Cecchetti (1997).
} 
tion in the calculation of the index. The 'CPI excluding food and energy' approach, for example, involves a simple re-weighting of individual prices of goods and services, excluding food and energy, to obtain an underlying measure. As argued by Bryan and Cecchetti (1994), the weighted median, as one of trimmed means, is able to reduce noise in a number of ways, such as to downweight the importance of sector-specific shocks likely to only eventually average to zero across all prices and to reduce the impact of errors in price setting or measurement.

\section{Data and methods}

The data set used in this paper includes the year-ended and quarterly rates of the following measures of inflation in Australia: the all groups CPI, the all groups CPI excluding volatile items (fruit, vegetables and automotive fuel), the measure of market prices excluding volatile items, the weighted median and the trimmed mean. The first one is the headline rate and the rest are underlying measures. The data set is obtained from the Reserve Bank of Australia web site (www.rba.gov.au) and covers the 1976-2003 period. ${ }^{5}$

As suggested above, the long-term trend in inflation $\left(\Pi_{t}\right)$ can be approximated by a moving average of inflation rates. Similar to Cecchetti (1997), this paper uses a 13quarter centred moving average of actual inflation (the quarterly headline rate), and as a result the effective sample period becomes 1977Q3-2001Q4. ${ }^{6}$ The choice of a 3 year window is not critical and this choice can also be compared to the choices made in Kearns (1998) (5,7 and 9 quarters). ${ }^{7}$

The ability of various indicators of inflation to reduce noise are evaluated by their accuracy in predicting this long-term trend in inflation. The question of accuracy is

\footnotetext{
${ }^{5}$ The sample period for the CPI excluding volatile items and the measure of market prices excluding volatile items is 1987Q1-2003Q2.

${ }^{6}$ It seems that the seasonality of Australian inflation data is not strong as the difference between seasonal-adjusted rates and non-adjusted rates is very small. The tests for the presence of seasonality in the data are not significant.

${ }^{7}$ Changing to a 15 -quarter centred moving average does not change the qualitative results reported in the paper.
} 
Figure 1: Selected inflation measures and long-term trend, per cent per annum

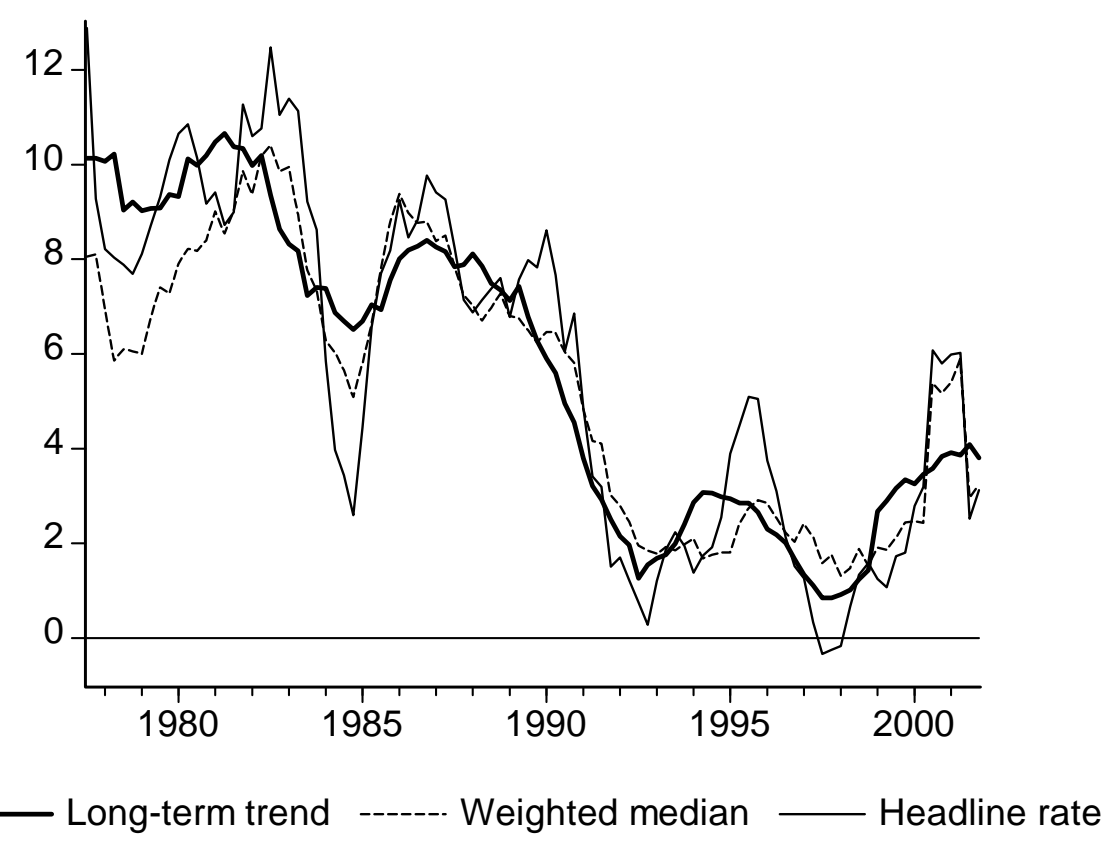

examined in two ways, predictive accuracy and directional accuracy. The first one answers the question of whether indicators tend to be near the long-term trend and the second one answers the question of whether they tend to correctly predict the direction of any change in the inflation trend. Accuracy can only be judged relatively, and moreover measurements of accuracy do not offer guidance on how to improve indicators.

Figure 1 plots the long-term trend in inflation (i.e., the annualised rate of the 13quarter centred moving average), as well as two year-ended rates: the headline rate and the weighted median. It is clear that the headline rate is more volatile than the underlying measures, such as the weighted median. Since 1993, however, the volatility of the headline rate seems to become smaller than before. The relative accuracy of the headline rate and underlying measures in predicting the long-term trend in inflation can be tested by formal statistical tests.

The paper performs the Diebold-Mariano (DM) (1995) test of predictive accuracy between the indicators. Let $\widehat{y_{1} t}$ and $\widehat{y_{2} t}$ be two predictions of the long-term trend in 
inflation $y_{t}$, and $e_{1 t}$ and $e_{2 t}$ be the associated predictive errors. If $g\left(e_{i t}\right)$ is a direct function of the predictive errors and $d_{t}=g\left(e_{1 t}\right)-g\left(e_{2 t}\right)$, then the equality of predictive accuracy can be tested by the following statistic

$$
S_{1}=\frac{\bar{d}}{\sqrt{V(\bar{d})}}
$$

where $\bar{d}$ is the mean of $d$, and $V(\bar{d})$ is the variance of $\bar{d}$. Asymptotically, a consistent estimate of $V(\bar{d})$ is

$$
V(\bar{d}) \approx \frac{\gamma_{0}+2 \sum_{k=1}^{h} \gamma_{k}}{T}
$$

where $h$ is the truncation lag, and $\gamma_{k}$ is the $k$ th autocovariance of $d_{t}$ and can be estimated by

$$
\gamma_{k}=\frac{\sum_{t=k+1}^{T}\left(d_{t}-\bar{d}\right)\left(d_{t-k}-\bar{d}\right)}{T}
$$

The truncation lag is often related to a familiar result that optimal $(h+1)$-step-ahead prediction errors are at most $h$-dependent, implying all the sample autocovariances beyond $h$ are zero. The sample autocorrelation function of $d_{t}$ can also be used to determine the truncation lag.

Harvey, Leybourne and Newbold (1997) note that the Diebold-Mariano test could be seriously over-sized as the prediction horizon, $h$, increases, therefore they provide a modified Diebold-Mariano test statistic

$$
S_{1}^{*}=S_{1} \sqrt{\frac{T+1-2(h+1)+h(h+1) / T}{T}}
$$

where $S_{1}$ is the original statistic (3). Harvey, Leybourne and Newbold also recommend a further modification of comparing the statistics with critical values from the Student's $t$ distribution with $(T-1)$ degrees of freedom, rather than from the standard normal distribution.

The analysis of directional accuracy is to test the null hypothesis that the probability of correctly predicting the moving direction of the long-term trend in the two consecutive periods is the same for two indicators. The numbers of correct and incorrect predictions 
by two indicators are tallied in a contingency table, which are then used to construct two alternative test statistics, a chi-squared test and a more conservative statistic introduced by Yates $(1934){ }^{8}$

\section{Relative accuracy of underlying measures}

This section analyses the relative accuracy of the underlying measures by applying the methods described in the previous section. All the quarterly rates of underlying measures will be compared with the quarterly headline rate, while the year-ended rates of underlying measures will be compared with the year-ended headline rate. The longterm trend in inflation, which is approximated by the 13-quarter centred moving average of the quarterly headline rate, is annualised in the comparison of the year-ended rates. This section reports the results of predictive accuracy first, then those of directional accuracy.

For predictive accuracy, two direct functions of errors are used in this paper: absolute error, $g(e)=|e|$, and mean squared error, $g(e)=e^{2}$. Since the long-term trend includes the information of future inflation in the next six quarters, all the inflation indicators are implicitly six-step-ahead forecasts. The arguments in the previous section suggest the need to allow for at least five-dependent predictive errors. This intuition is confirmed by the sample autocorrelation functions, and the related Ljung-Box Q-statistics, of the loss differentials, in which sizable and significant sample autocorrelations appear at lags up to five, and nowhere else.

Table 1 presents the test results for the quarterly rates, while those for the year-ended rates are reported in Table 2. These tables also report the results for the sub-period since 1993Q1, when the Reserve Bank of Australia formally adopted inflation targeting. ${ }^{9}$

The test results in Table 1 clearly show that the underlying measures outperform the headline rate in predicting the long-term trend in inflation. All the forecast errors,

\footnotetext{
${ }^{8}$ A detailed explanation of these two test statistics can be found in Conover (1980)

${ }^{9}$ The truncation lag $h$ is chosen as 2 for all the tests conducted for this period. The sample autocorrelation functions and associated Ljung-Box Q-statistics confirm that sizable and significant sample autocorrelations appear at lag 1 and 2 and nowhere else.
} 
Table 1: Predictive accuracy relative to the headline rate: the quarterly rates

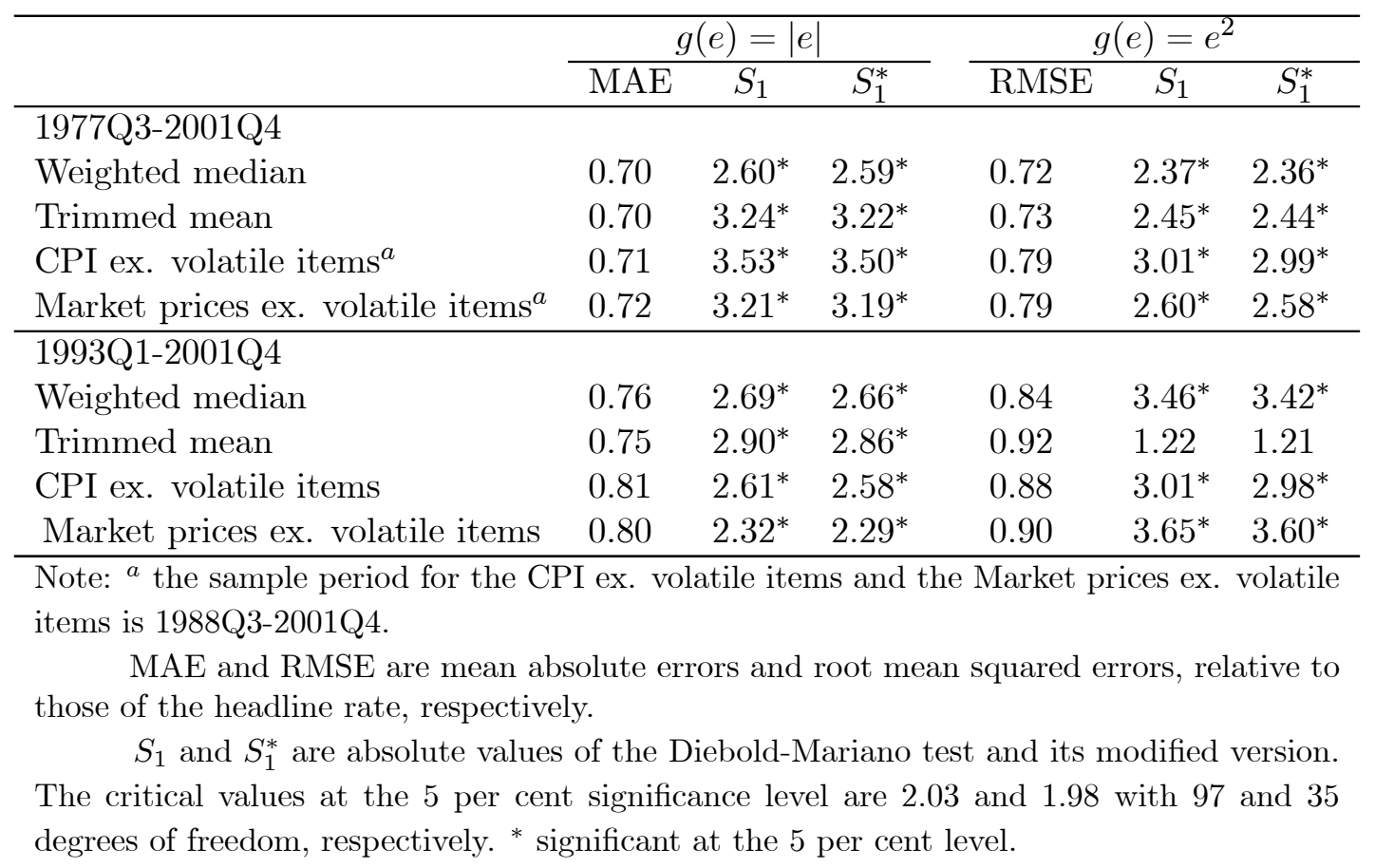

either absolute errors or squared errors, of the underlying measures are smaller than those of the headline rate in both sample periods, with almost all the test statistics are significant. As demonstrated in the previous section, the headline rate is a poor approximation of the long-term inflation trend and has much noise in it (see Equation 2). The underlying measures do have the ability to extract more information of future inflation from the price data than the headline rate.

When the year-ended rates of the underlying measures are compared to the yearended headline rate, however, the results are different. While the means of forecast errors, either absolute errors or squared errors, of the underlying measures are still smaller than those of the headline rate (but not as large as the quarterly rates), the test statistics cannot reject the null hypothesis of equal predictive accuracy overwhelmingly. The only exception is the measure of market prices excluding volatile items, which rejects the null in both sample periods. There is also weak evidence supporting the weighted median in the inflation targeting period, with the test statistics for root squared means errors significant at the 5 per cent level. 
Table 2: Predictive accuracy relative to the headline rate: the year-ended rates

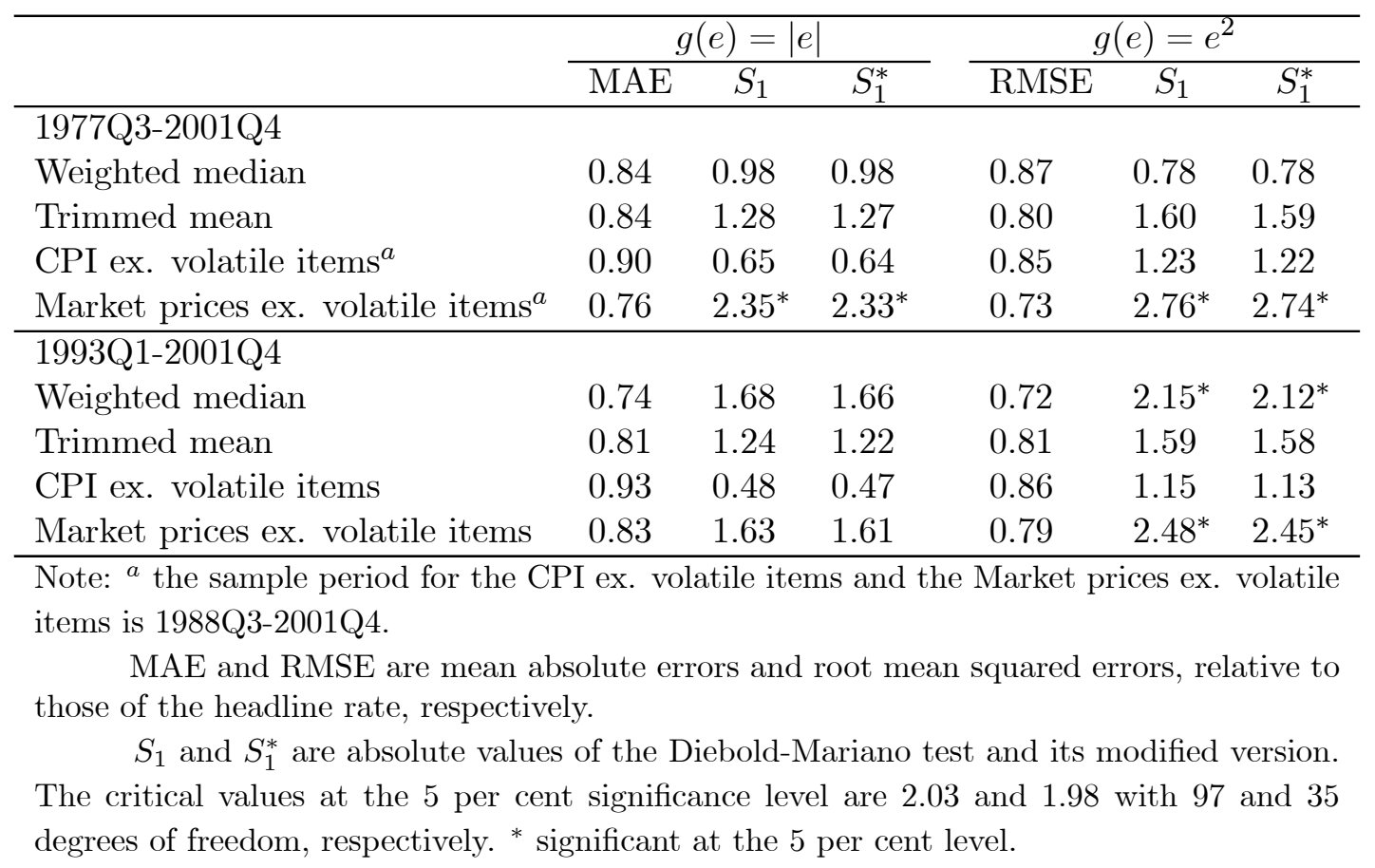

Year-ended rates are essentially an average of the quarterly rates over the year, therefore these year-ended rates are less volatile than the quarterly rates and may provide more information of the long-term trend. The year-ended headline rate is able to reduce the volatility in the quarterly rate, with a mean absolute error of 1.2 percentage points per annum from the long-term trend, much smaller than 1.8 percentage points for the quarterly headline rate. The results in Table 2 suggest that in terms of predictive accuracy, the year-ended rates of the underlying measures and the headline rate are not statistically different and they provide the same information of the long-term trend in inflation.

Similarly for directional accuracy, the quarterly and year-ended rates of the underlying measures are respectively compared to the corresponding headline rate, and the results are reported in Tables 3 and 4 . In every case, the probability of the headline rate correctly predicting the direction of the change in the long-term trend is about 60 per cent, higher than those of the underlying measures, but the statistical tests cannot reject the null hypothesis of no differences in the probability. This suggests that the 
Table 3: Directional accuracy relative to the headline rate: quarterly rates

\begin{tabular}{lccccc}
\hline Indicator and sample & Correct & Incorrect & $\begin{array}{c}\text { Percent } \\
\text { correct }\end{array}$ & Chi-Squared & Yates \\
\hline 1977Q3-2001Q4 & & & & & \\
Headline rate & 63 & 34 & 65 & & \\
Weighted median & 55 & 42 & 57 & 1.38 & 1.06 \\
Trimmed mean & 59 & 38 & 61 & 0.35 & 0.20 \\
CPI ex. volatile items $^{a}$ & 27 & 32 & 46 & 2.18 & 1.67 \\
Market prices ex. volatile items $^{a}$ & 31 & 28 & 53 & 0.55 & 0.31 \\
\hline 1993Q1-2001Q4 $^{\text {Headline rate }}$ & 20 & 15 & 57 & & \\
Weighted median & 16 & 19 & 46 & 0.92 & 0.51 \\
Trimmed mean & 16 & 19 & 46 & 0.92 & 0.51 \\
CPI ex. volatile items & 14 & 21 & 40 & 2.06 & 1.43 \\
Market prices ex. volatile items & 17 & 18 & 49 & 0.52 & 0.23 \\
\hline Not:
\end{tabular}

Note: ${ }^{a}$ the sample period for the CPI ex. volatile items and the Market prices ex. volatile items is 1988Q3-2001Q4.

The critical value at the 5 per cent significance level is $3.84 .{ }^{*}$ significant at the 5 per cent level.

The description of the tests can be found in Conover (1980, pp.144-151).

Table 4: Directional accuracy relative to the headline rate: year-ended rates

\begin{tabular}{lccccc}
\hline Indicator and sample & Correct & Incorrect & $\begin{array}{c}\text { Percent } \\
\text { correct }\end{array}$ & Chi-Squared & Yates \\
\hline 1977Q3-2001Q4 & 58 & 39 & 60 & & \\
Headline rate & 55 & 42 & 57 & 0.19 & 0.08 \\
Weighted median & 59 & 38 & 61 & 0.02 & 0.00 \\
Trimmed mean & 30 & 26 & 54 & 0.14 & 0.04 \\
CPI ex. volatile items $^{a}$ & 31 & 25 & 55 & 0.04 & 0.00 \\
Market prices ex. volatile items $^{a}$ & & & & & \\
\hline 1993Q1-2001Q4 & 20 & 15 & 57 & & \\
Headline rate & 16 & 19 & 46 & 0.92 & 0.51 \\
Weighted median & 19 & 16 & 54 & 0.06 & 0.00 \\
Trimmed mean & 17 & 18 & 49 & 0.52 & 0.23 \\
CPI ex. volatile items & 20 & 15 & 57 & 0.00 & 0.00 \\
Market prices ex. volatile items & 20 & & & & \\
\hline Note: & & & & & \\
\hline
\end{tabular}

Note: ${ }^{a}$ the sample period for the CPI ex. volatile items and the Market prices ex. volatile items is 1988Q3-2001Q4.

The critical value at the 5 per cent significance level is $3.84 .{ }^{*}$ significant at the 5 per cent level.

The description of the tests can be found in Conover (1980, pp.144-151). 
underlying measures and the headline rates are not different in predicting directional changes in the long-term trend in inflation.

\section{Conclusion}

With the long-term trend in inflation approximated by the 13-quarter centred moving average of actual inflation, this paper has compared the accuracy of underlying measures of inflation relative to the headline rates. The comparison has been conducted in two areas: predictive and directional accuracy, and the standards of predictive quality are formally tested.

By using Australian data, it is found that while the underlying measures did outperform the quarterly headline rate, the predictive accuracy of the underlying measures is not significantly different from that of the year-ended headline rate. The only exception is the measure of market prices excluding volatile items, which has significant test results in the whole sample period 1977-2001 as well as in the inflation targeting sub-period starting from 1993. There is also weak evidence of the weighted median outperforming the year-ended headline rate in the inflation targeting period. As regards directional accuracy, though the headline rates had a higher probability of correctly predicting directional changes in the long-term trend than the underlying measures, the test results are not statistically significant.

With no clear and overwhelming evidence of the underlying measures outperforming the year-ended headline rate in the Australian data, the Reserve Bank of Australia should not rely only on the underlying measures of inflation as the headline rate is equally important in predicting the long-term trend in inflation. Moreover, since the existing measures are not satisfactory (mean absolute errors of about 1 percentage point from the long-term trend), it may be necessary to explore other approaches to measuring the long-term trend, which can be statistical measures, such as the dynamic factor index (DFI) measure proposed by Bryan and Cecchetti (1993), or based on economic models, such as those suggested by Quah and Vahey (1995) and Stock and Watson (1999). 


\section{References}

Boskin, M. J. and et al. (1997), 'The CPI Commission: Findings and Recommendations,' American Economic Review 87(2), 78-83.

Bryan, M. F. and S. G. Cecchetti (1993), 'The Consumer Price Index as a Measure of Inflation,' Economic Review of the Federal Reserve Bank of Cleveland 29(4), 15-24.

- (1994), 'Measuring Core Inflation,' in Monetary Policy, ed. by N. G. Mankiw, Studies in Business Cycles, vol. 29, University of Chicago Press, Chicago, 195-215.

Cecchetti, S. G. (1997), 'Measuring Short-Run Inflation for Central Bankers,' Federal Reserve Bank of St. Louis Review 79(3), 143-55.

Cecchetti, S. G. and E. L. Groshen (2000), 'Understanding Inflation: Implications for Monetary Policy,' Working paper 7482, NBER.

Conover, W. J. (1980), Practical Nonparametric Statistics, Wiley series in probability and mathematical statistics., Wiley, New York, 2nd edn.

Diebold, F. X. and R. S. Mariano (1995), 'Comparing Predictive Accuracy,' Journal of Business and Economic Statistics 13(3), 253-63.

Dixon, R. and G. C. Lim (2003), 'Underlying Inflation in Australia: Are the Existing Measures Satisfactory?,' unpublished manuscript, The University of Melbourne.

Freeman, D. G. (1998), 'Do Core Inflation Measures Help Forecast Inflation?,' Economics Letters 58(2), 143-47.

Harvey, D., S. Leybourne and P. Newbold (1997), 'Testing the Equality of Prediction Mean Squared Errors,' International Journal of Forecasting 13(2), 281-91.

Kearns, J. (1998), 'The Distribution and Measurement of Inflation,' Research Discussion Paper 9810, Reserve Bank of Australia.

Le Bihan, H. and F. Sedillot (2000), 'Do Core Inflation Measures Help Forecast Inflation? Out-of-Sample Evidence from French Data,' Economics Letters 69(3), 261-66. 
Quah, D. and S. P. Vahey (1995), 'Measuring Core Inflation,' Economic Journal 105(432), 1130-44.

Reserve Bank of Australia (2002), 'Statement of Monetary Policy,' Reserve Bank of Australia Bulletin (May), 1-56.

Stock, J. H. and M. W. Watson (1999), 'Forecasting Inflation,' Journal of Monetary Economics 44(2), 293-335.

Wynne, M. A. (1999), 'Core Inflation: A Review of Some Conceptual Issues,' Working Papers 9903, Federal Reserve Bank of Dallas.

Yates, F. (1934), 'Contingency Tables Involving Small Numbers and the $\chi^{2}$ Test,' Journal of the Royal Statistical Society 1, 217-35. 


\section{University Library}

\section{- M M N E R VA A gateway to Melbourne's research publications}

Minerva Access is the Institutional Repository of The University of Melbourne

Author/s:

Song, Lei Lei

Title:

Do Underlying Measures of Inflation Outperform Headline Rates? Evidence from Australian Data

Date:

2003-12

Citation:

Song, Lei Lei (2003) Do Underlying Measures of Inflation Outperform Headline Rates? Evidence from Australian Data.

Persistent Link:

http://hdl.handle.net/11343/33801 